\title{
Two-Dimensional Fluorinated Boron Sheets: Mechanical, Electronic, and Thermal Properties
}

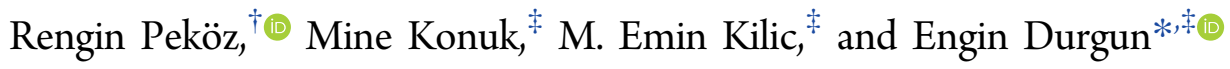 \\ ${ }^{\dagger}$ Department of Electrical and Electronics Engineering, Attlım University, 06836 Ankara, Turkey \\ ${ }^{\ddagger}$ UNAM-National Nanotechnology Research Center and Institute of Materials Science and Nanotechnology, Bilkent University, \\ Ankara 06800, Turkey
}

Supporting Information

ABSTRACT: The synthesis of atomically thin boron sheets on a silver substrate opened a new area in the field of two-dimensional systems. Similar to hydrogenated and halogenated graphene, the uniform coating of borophene with fluorine atoms can lead to new derivatives of borophene with novel properties. In this respect, we explore the possible structures of fluorinated borophene for varying levels of coverage $\left(\mathrm{B}_{n} \mathrm{~F}\right)$ by using first-principles methods. Following the structural optimizations, phonon spectrum analysis and ab initio molecular dynamics simulations are performed to reveal the stability of the obtained structures. Our results indicate that while fully fluorinated borophene $(\mathrm{BF})$ cannot be obtained, stable configurations with lower coverage levels $\left(\mathrm{B}_{4} \mathrm{~F}\right.$ and $\left.\mathrm{B}_{2} \mathrm{~F}\right)$

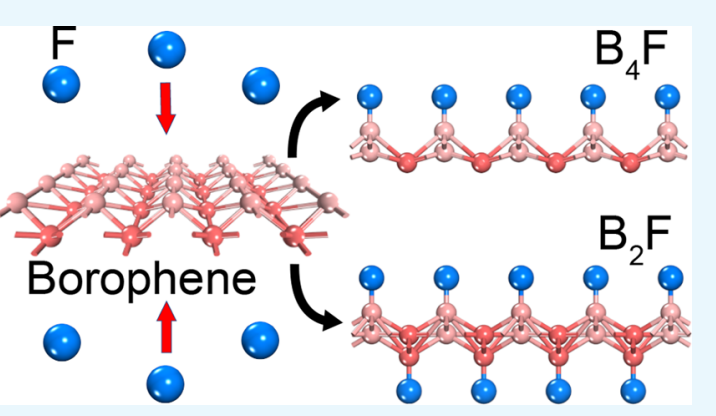
can be attained. Unveiling the stable structures, we explore the mechanical, electronic, and thermal properties of $\left(\mathrm{B}_{n} \mathrm{~F}\right)$. Fluorination significantly alters the mechanical properties of the system, and remarkable results, including direction-dependent variation of Young's modulus and a switch from a negative to positive Poisson's ratio, are obtained. However, the metallic character is preserved for low coverage levels, and metal to semiconductor transition is obtained for $\mathrm{B}_{2} \mathrm{~F}$. The heat capacity at a low temperature increases with an increasing $\mathrm{F}$ atom amount but converges to the same limiting value at high temperatures. The enhanced stability and unique properties of fluorinated borophene make it a promising material for various high-technology applications in reduced dimensions.

\section{INTRODUCTION}

Following the realization of graphene and revealing its novel mechanical, electronic, and optical properties, ${ }^{1}$ great amounts of theoretical and experimental studies have been devoted to investigate alternative two-dimensional (2D) materials. ${ }^{2}$ In this quest, various $2 \mathrm{D}$ monoelemental structures beyond graphene (such as silicene, ${ }^{3,4}$ germanene, ${ }^{5}$ stanene, ${ }^{6}$ phosphorene, ${ }^{7}$ antimonene, ${ }^{8}$ and borophene ${ }^{9,10}$ ) have been realized. The main focus of these studies is not only to explore new systems but also to integrate these materials into next-generation nanoscale applications and to enhance the performance of these devices by tuning their intrinsic properties.

Among 2D monoelemental structures, the recent synthesis of an atomically thin boron sheet (i.e., borophene) on an $\operatorname{Ag}(111)$ substrate with a buckled triangular structure ${ }^{9}$ has aroused increasing interest because of its exceptional properties. Contrary to all-known bulk boron allotropes that are semiconductors at ambient conditions, borophene presents metallic behavior with strong anisotropy. ${ }^{9}$ Furthermore, buckled borophene has been predicted to display novel mechanical properties. As an example, the Young's modulus is reported to be $170 \mathrm{GPa} \cdot \mathrm{nm}$ along the corrugated direction and $398 \mathrm{GPa} \cdot \mathrm{nm}$ along the uncorrugated direction, ${ }^{9}$ which is even higher than that of graphene $(340 \mathrm{GPa} \cdot \mathrm{nm}){ }^{11}$ The system also has a negative Poisson's ratio because of the out-of-plane buckling. ${ }^{9}$ A theoretical investigation on thermal properties indicates that the thermal conductivity of borophene is also anisotropic and low because of the strong phonon-phonon scattering, and negative thermal expansion coefficients are obtained for both directions. ${ }^{12,13}$ In a computational study, large optical anisotropy with high optical transparency is reported. $^{14}$

In addition to the buckled phase, planar boron sheets with periodic holes are also grown epitaxially on an $\operatorname{Ag}(111)$ substrate, ${ }^{10}$ and various stable phases with novel properties are theoretically predicted. ${ }^{15-18}$ A comprehensive search on the possible structures indicates that orthorhombic $2 \mathrm{D}$ phases of boron can have a distorted Dirac cone and possess massless Dirac fermions with strongly direction-dependent quasiparticle group velocities. ${ }^{19}$ Borophene sheets can also display an intrinsic superconducting behavior at low temperatures ${ }^{15,20}$ which can be modified by strains and dopings. ${ }^{21}$ The anisotropic high bending flexibility makes borophene an ideal candidate for fabricating flexible electronic devices. ${ }^{22}$ These extraordinary properties suggest borophene as a promising material for various technological applications. ${ }^{23-25}$

Received: November 6, 2017

Accepted: January 26, 2018

Published: February 12, 2018 
Chemical modification, particularly hydrogenation, is one of the well-known methods to tailor the intrinsic properties of $2 \mathrm{D}$ materials and even new derivatives can be formed. ${ }^{26-29}$ Besides hydrogenation, halogenation (especially with fluorine atoms) is another way of coating $2 \mathrm{D}$ materials because of their high electronegativity. Therefore, structural, electronic, and mechanical properties of halogenated graphene, ${ }^{30-33}$ silicene, ${ }^{34}$ germanene, ${ }^{35,36}$ arsenene, ${ }^{37}$ and $\operatorname{tin}^{38}$ have been investigated in detail. Fully fluorinated graphene (fluorographene, CF) has been synthesized and found that $\mathrm{CF}$ is a high-quality insulator with an optical gap of $\sim 3 \mathrm{eV}^{39}$ Furthermore, it has high thermal and chemical stabilities and reported to be mechanically stiff but stretchable. ${ }^{39}$ Bilayer fluorinated graphene is investigated theoretically as well and compared with graphane and fluorographene. ${ }^{40}$ In addition to graphene, recently, the effect of halogenation on the electronic properties of silicene is studied and a band gap opening upon halogenation is reported. ${ }^{34}$ For germanene, the interaction with halogen atoms enhances the spin-orbit coupling and quantum spin hall effect can be realized at ambient conditions. ${ }^{35}$ For the diatomic 2D structures, chlorination of AlN nanosheets is studied based on first-principles methods, and it is shown that half-chlorination (saturation of only $\mathrm{Al}$ sites) converts the semiconducting system into a half-metallic ferromagnet. ${ }^{41}$ Furthermore, although $2 \mathrm{D} \mathrm{BN}$ is an insulator, decoration with fluorine can make it a ferromagnetic half-metal or an antiferromagnetic semiconductor with a narrow band gap. ${ }^{42}$

As in-plane bonds resulting from $\mathrm{sp}^{2}$ hybridizations are in general stronger than the out-of-plane bonds, buckled triangular borophene is less stable than planar porous borophene polymorphs where two- and three-center bondings are balanced. Therefore, the interaction with halogen atoms not only modifies the intrinsic properties of borophene but can also enhances the stability by the occupation of out-of-plane bonding states. Despite its importance, to the best of our knowledge, only the interaction of single halogen atoms/ molecules with borophene is studied, ${ }^{43}$ and halogenation has not been explored yet. With this motivation, in this work, we investigate the possible fluorinated derivatives of borophene and analyze the mechanical, electronic, and thermal properties of the stable systems by using ab initio methods. Starting from the interaction of a single $\mathrm{F}$ atom with borophene, we study $\mathrm{B}_{n} \mathrm{~F}(n=1,2$, and 4$)$ structures with single- and double-sided coverages and consider various possible geometries. The level of coverage is defined by $\Theta(\%)=1 / n$, referring to the ratio of one $\mathrm{F}$ atom to $n \mathrm{C}$ atoms in percentage. The stability of the obtained structures is tested by phonon spectrum analyses and high-temperature ab initio molecular dynamics (AIMD) simulations. While $\mathrm{B}_{4} \mathrm{~F}$ and $\mathrm{B}_{2} \mathrm{~F}$ systems result in stable configurations, fully fluorinated borophene $(\mathrm{BF})$ is found to be unstable at ambient conditions. The mechanical properties of thermodynamically stable systems are analyzed, and remarkable results, including a drastic change in Young's modulus and a switch from a negative to positive Poisson's ratio, are obtained. Additionally, electronic structure calculations indicate a metal to semiconductor transition at a high level of coverage. Finally, thermal properties are explored, and the variations of heat capacity and entropy with $\Theta$ are revealed.

\section{RESULTS AND DISCUSSIONS}

Atomic Structure and Stability. We start with the structural optimization of monolayer-buckled borophene (i.e., borophene) which is shown in Figure 1a. The calculated lattice
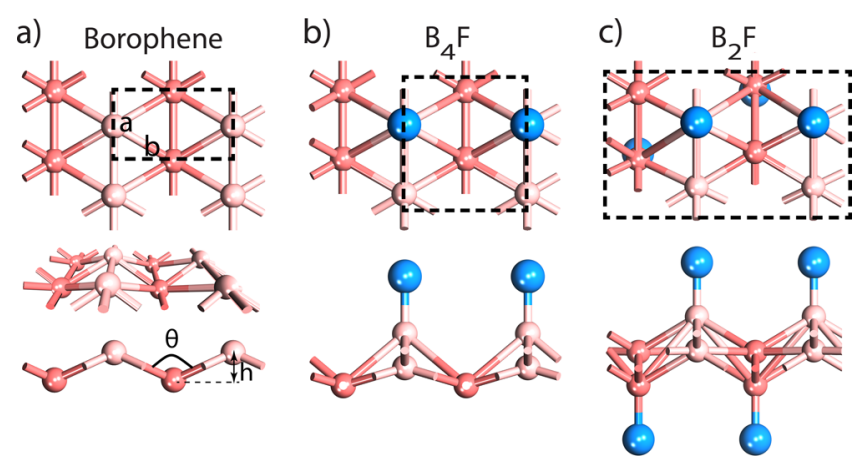

Figure 1. Top (upper panel) and side (lower panel) views of optimized ground-state structures of (a) bare borophene, (b) $\mathrm{B}_{4} \mathrm{~F}$, single-sided fluorination of borophene with $\Theta=25 \%$ (level of coverage), and (c) $B_{2} F$, double-sided fluorination of borophene with $\Theta$ $=50 \%$. The unit cells are framed by the black dashed lines. Dark and light pink represent boron atoms which are on the downward and upward planes, respectively, and blue represents F atoms. Lattice constants $(\mathrm{a}, \mathrm{b})$, dihedral angle $(\theta)$, and the buckling height $(h)$ are shown.

constants of borophene are $|\mathbf{a}|=1.62 \AA$ and $|\mathbf{b}|=2.87 \AA$, which are in good agreement with the previous experimental and theoretical results. ${ }^{9,14,44}$ Borophene has a buckled triangular structure where the adjacent rows of boron atoms are alternately raised up or lowered down (along b) with a buckling height, $h=0.91 \AA$. The $\mathrm{B}-\mathrm{B}$ bond length along $\mathbf{b}\left(d_{\mathrm{B}-\mathrm{B}}^{\mathrm{b}}\right)$ is $1.87 \AA$, whereas it is $1.62 \AA$ along $\mathbf{a}\left(d_{\mathrm{B}-\mathrm{B}}^{\mathrm{a}}\right)$. The bond angle $\theta=100.1^{\circ}$ indicates that the atomic orbitals of borophene possess $\mathrm{sp}^{3}$-like hybridization to enhance the stabilization. High $h$ induces strong anisotropy, ${ }^{14,28,45}$ and the corrugated structure makes the surface chemically reactive. Some of the in-plane antibonding states of borophene are occupied, which tends the system to donate electrons and also induces structural instability. ${ }^{46,47}$

Before investigating the fluorination of borophene, we first examine the interaction of a single $\mathrm{F}$ atom with borophene. Understanding the adsorption process of a single atom is crucial to reveal the fluorinated derivates. In our previous study, by taking a large $7 \times 4$ supercell (with lattice parameters of 11.30 and $11.45 \AA$ ), we showed that F strongly binds to the borophene surface and the top of the $\mathrm{B}$ atom on the upper layer (T-site) is the most favorable adsorption site. ${ }^{43}$ The obtained energy barriers along the diffusion paths are very high $(1.26 \mathrm{eV}$ along a and $0.84 \mathrm{eV}$ along $\mathbf{b}$ ), indicating low possibility of migration. Moreover, $\mathrm{F}_{2}$ dissociates spontaneously without an activation barrier on the borophene surface, suggesting that adsorption of $\mathrm{F}$ is an exothermic process. ${ }^{43}$ Here, we repeat these calculations for a $2 \times 2$ supercell $\left(\mathrm{B}_{8} \mathrm{~F}\right.$ with $\left.\Theta=12.5 \%\right)$ where $\mathrm{F}-\mathrm{F}$ interactions become significant. Considering the symmetry of borophene, five different $\mathrm{F}$ adsorption sites are investigated and presented in Figure S1 (Supporting Information). Similar to single-atom adsorption, an $\mathrm{F}$ atom always prefers the T-site. The $E_{\mathrm{b}}$ is calculated as $5.42 \mathrm{eV}$, and the increase in $E_{\mathrm{b}}$ with respect to single $\mathrm{F}$ adsorption indicates an attractive interaction between the $F$ atoms at this level of coverage. We further investigate the possible adsorption sites for two $F$ (with $\Theta=6.25 \%$ ) and four $F$ atoms (with $\Theta=$ $12.5 \%)$ in a $4 \times 4$ supercell. We find that increasing the number of $\mathrm{F}$ atoms does not change the adsorption site of $\mathrm{F}$. The resulting structures and obtained $E_{\mathrm{b}}$ values are summarized in Figures S2-S4 and Table S1 (Supporting Information). 
Table 1. Structural Properties and Energetics of Bare and Fluorinated Derivatives of Borophene ${ }^{a}$

\begin{tabular}{lccccccccccc}
\multicolumn{1}{c}{ structure } & unit cell & $a(\AA)$ & $b(\AA)$ & $h(\AA)$ & $\Theta(\%)$ & $E_{\mathrm{b}}(\mathrm{eV})$ & $E_{\mathrm{f}}(\mathrm{eV})$ & $\rho_{\mathrm{F}}(|e|)$ & $d_{\mathrm{B}-\mathrm{F}}(\AA)$ & $d_{\mathrm{B}-\mathrm{B}}^{\mathrm{a}}(\AA)$ & \\
borophene & $1 \times 1$ & 1.62 & 2.87 & 0.91 & & & & & 1.62 \\
$\mathrm{~B}_{4} \mathrm{~F}(\mathrm{~s})$ & $2 \times 1$ & 3.26 & 2.90 & 1.21 & 25 & 5.31 & 3.97 & 0.81 & 1.35 & 1.82 \\
$\mathrm{~B}_{2} \mathrm{~F}(\mathrm{~d})$ & $2 \times 2$ & 3.20 & 5.80 & 1.70 & 50 & 5.02 & 3.68 & 0.78 & 1.35 & $1.75-1.84$ & $1.68-1.82-2.41$
\end{tabular}

${ }^{a}$ Structure, lattice constants ( $a$ and $b$ in $\AA$ ), buckling height ( $h$ in $\AA$ ), level of coverage $\left(\Theta\right.$ in \%), binding energy $\left(E_{\mathrm{b}}\right.$ in eV/F atom), formation energy $\left(E_{\mathrm{f}}\right.$ in eV/F atom), charge transferred to $\mathrm{F}$ atoms $\rho_{\mathrm{F}}(|e|), \mathrm{B}-\mathrm{F}$ bond length $\left(d_{\mathrm{B}-\mathrm{F}}\right.$ in $\AA$ ), and $\mathrm{B}-\mathrm{B}$ bond lengths along a and $\mathbf{b}\left(d_{\mathrm{B}-\mathrm{B}}^{\mathrm{b}}\right.$ and $d_{\mathrm{B}-\mathrm{B}}^{\mathrm{b}}$ in $\AA$ ) are listed. "s" and "d" in parenthesis indicate single- and double-sided coverages, respectively. Only the results for stable configurations are reported.

Accordingly, for high coverage levels, we only consider the Tsite for the adsorption of $\mathrm{F}$ atoms.

Inspired by the studies on hydrogenation of borophene ${ }^{46,48-50}$ (i.e., borophane) and using the $F$ atom adsorption results, we increase the level of $\mathrm{F}$ coverage gradually and investigate the structural properties and energetics of $\mathrm{B}_{n} \mathrm{~F}$. The obtained results are summarized in Table 1 . We first consider $\mathrm{B}_{4} \mathrm{~F}(\mathrm{~s})$, single-sided fluorination of borophene with $\Theta$ $=25 \%$. One-sided coverage corresponds to the case when borophene is on a substrate or on another $2 \mathrm{D}$ system. Among the possible geometries, the lowest energy and stable (see below) configuration are obtained and presented in Figure $1 \mathrm{~b}$. In this case, the $\mathrm{F}$ atoms are located on top of alternating $\mathrm{B}$ atoms along both directions. Upon relaxation, its $h$ increases by $0.3 \AA$ compared to the pristine borophene and $d_{\mathrm{B}-\mathrm{F}}$ is calculated as $1.35 \AA$. The attractive interaction between $F$ atoms increases $E_{\mathrm{b}}$ when compared to the cases with lower $\Theta$. We also try the possibility of $\mathrm{B}_{2} \mathrm{~F}(\mathrm{~s})$ by attaching $\mathrm{F}$ atoms to each $\mathrm{B}$ atom on the upper layer (see Figure S5, Supporting Information for initial configurations). It is found that the calculated $E_{\mathrm{b}}$ reduced down to $1.32 \mathrm{eV}$ and also the structure is significantly distorted because of a strong repulsive $\mathrm{F}-\mathrm{F}$ interaction. Thus, a stable $\mathrm{B}_{2} \mathrm{~F}(\mathrm{~s})$ structure with $\Theta=50 \%$ cannot be obtained.

As a next step, we investigate $\mathrm{B}_{2} \mathrm{~F}(\mathrm{~d})$, the double-sided fluorination of borophene with $\Theta=50 \%$, as presented in Figure 1c. The armchair B-B bonds are weakened in this configuration, and $d_{\mathrm{B}-\mathrm{B}}$ becomes nonuniform and increases to 1.75-1.84 $\AA$. The situation for $d_{\mathrm{B}-\mathrm{B}}^{\mathrm{b}}$ is more complicated, and it increases (decreases) for $B$ atoms with (without) $F$. Furthermore, $h$ becomes $1.70 \AA$, which is significantly larger than that of bare borophene, resulting from the considerable modification of $\mathrm{B}-\mathrm{B}$ bonds. The $d_{\mathrm{B}-\mathrm{F}}$ is calculated as $1.35 \AA$, which remained the same as $\mathrm{B}_{4} \mathrm{~F}(\mathrm{~s})$. The $E_{\mathrm{b}}$ reduced to $5.02 \mathrm{eV}$ but still high, indicating a strong binding.

Finally, double-sided full fluorination of borophene (BF) similar to the proposed structure of borophane $(\mathrm{BH})^{46,49}$ is investigated and three possible initial configurations are presented in Figure S6 (Supporting Information). BH is a stable configuration ${ }^{46}$ with each boron atoms being passivated by hydrogen atoms, resulting in a full coverage $(\Theta=100 \%)$. The hydrogenation of borophene has remarkably increased the lattice constant along a by $0.26 \AA \AA^{46}$ compared to the pristine structure. ${ }^{9}$ Accordingly, $d_{\mathrm{B}-\mathrm{B}}^{\mathrm{a}}$ in borophane is significantly stretched $(0.32 \AA)$, whereas $h$ is almost preserved. ${ }^{46}$ Contrary to $\mathrm{BH}$, the optimized configurations for $\mathrm{BF}$ cannot be obtained. Either the structure of borophene is drastically distorted or the $\mathrm{F}$ atoms form a cluster and do not bind to $\mathrm{B}$ atoms. Our results indicate that $d_{\mathrm{B}-\mathrm{B}}^{\mathrm{a}}$ is too small to accommodate $\mathrm{F}$ atoms on each $B$ atom, and the repulsive $F-F$ interaction at this level of coverage induces instability in this geometry. As a final effort, considering the lattice expansion in $\mathrm{BH}$ and the possibility of a structural phase transformation upon fluorination, we consider a $2 \times 2$ supercell (to remove the possible constraints) and gradually increase the lattice constants while optimizing for each case. Interestingly, we notice two geometric transitions upon expansion along the uncorrugated direction, and two possible BF phases are obtained. In the first phase, namely, a buckled honeycomb structure is obtained at $|\mathbf{a}|=4.63 \AA$ and $|\mathbf{b}|$ $=5.72 \AA . . d_{\mathrm{B}-\mathrm{B}}$ becomes almost uniform along both directions and calculated as $1.86 \AA$. The $h$ slightly increases and becomes $0.93 \AA$. The details of the transition are presented in Figure S7 (Supporting Information). In the second phase, namely, a buckled rectangular (almost square) structure (Figure S11, Supporting Information) is obtained at $|\mathbf{a}|=5.23 \AA$ and $|\mathbf{b}|=$ $4.76 \AA$. $d_{\mathrm{B}-\mathrm{B}}$ also becomes uniform and elongates to $1.97 \AA$, whereas $h$ decreases and measured as $0.86 \AA$. Last, it should be noted that $E_{\mathrm{f}}>0$ for all considered cases, revealing that fluorination with varying $\Theta$ is an exothermic process and can be achieved without an energy barrier.

Even though the structural optimization and binding (or formation) energies give an idea about the possible $\mathrm{B}_{n} \mathrm{~F}$ structures, they do not guarantee the thermodynamic stability. In this respect, we analyzed the phonon spectra of optimized $\mathrm{B}_{n} \mathrm{~F}$ systems, which are the candidate-fluorinated derivatives of borophene. The phonon dispersions of stable configurations including the pristine borophene are shown in Figure 2. The unit cell of borophene contains only two atoms; hence, its phonon spectrum is characterized by three acoustic and three optical phonon branches. The acoustic branches are the in-
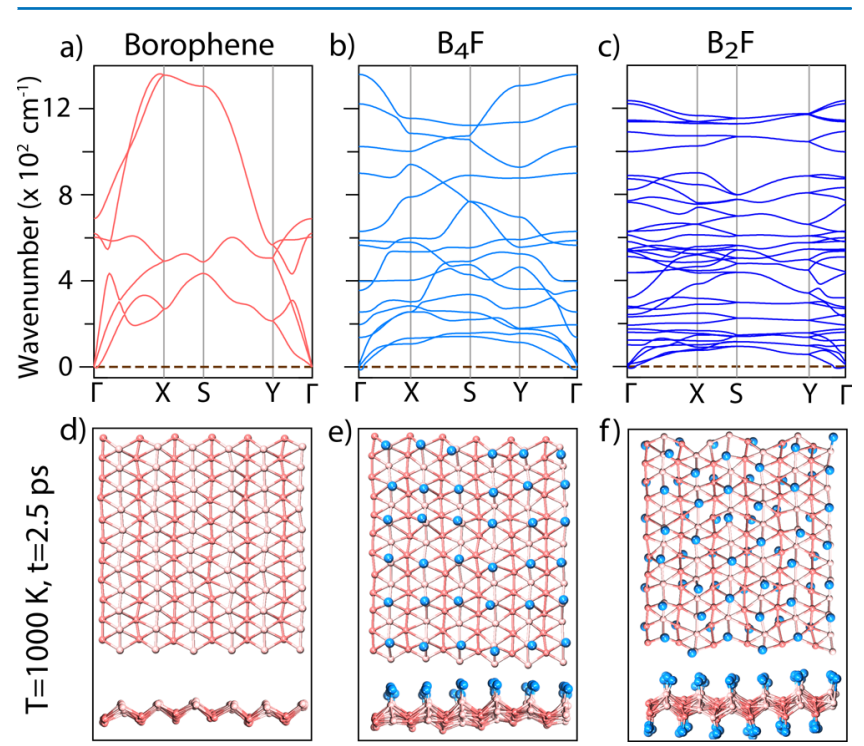

Figure 2. Calculated phonon dispersion curves and atomic configurations for AIMD simulations at $1000 \mathrm{~K}(\mathrm{a}-\mathrm{d})$ bare borophene, $(b-e) B_{4} F$, and $(c-f) B_{2} F$. Lower panels show the snapshots of the atomic configurations for AIMD simulations at 1000 $\mathrm{K}$ for the related structures. 
Table 2. Calculated Elastic Constants $\left(c_{i j}\right)$, Shear Modulus $(G)$, Young's Modulus $(Y$ in N/m), and Poisson's Ratios $(\nu)$ of Bare Borophene, $\mathrm{B}_{4} \mathrm{~F}(\mathrm{~s})$, and $\mathrm{B}_{2}(\mathrm{~d})$

$\begin{array}{lcccccccc}\text { structure } & c_{11} & c_{22} & c_{12} & c_{66}=G & Y_{a}(\mathrm{~N} / \mathrm{m}) & Y_{b}(\mathrm{~N} / \mathrm{m}) & \nu_{a} \\ \text { borophene } & 376.60 & 149.60 & -7.64 & 83.61 & 376.21 & 149.45 & -0.05 \\ \mathrm{~B}_{4} \mathrm{~F}(\mathrm{~s}) & 275.06 & 183.66 & 13.07 & 79.63 & 274.13 & 183.04 \\ \mathrm{~B}_{2} \mathrm{~F}(\mathrm{~d}) & 130.12 & 200.76 & 6.17 & 57.35 & 129.93 & 0.07 & 0.05 \\ \end{array}$

plane longitudinal acoustic, the transverse acoustic, and the outof-plane acoustic (ZA) modes. As seen in Figure 2a, there is a small imaginary frequency in ZA along the $\Gamma-X$ direction, which implies that the free-standing borophene is prone to instability against long-wavelength transversal waves, which clarifies the stripe formation along the armchair direction in the synthesis of borophene. ${ }^{9}$

The phonon dispersion curves of $\mathrm{B}_{4} \mathrm{~F}(\mathrm{~s})$ and $\mathrm{B}_{2} \mathrm{~F}(\mathrm{~d})$ are illustrated in Figure $2 \mathrm{~b}, \mathrm{c}$ for $2 \times 1$ and $2 \times 2$ unit cells, respectively. All frequencies are positive excepting the lowest ZA mode which exhibits a slight softening around $\Gamma$-point. The absence of phonon modes with a negative frequency suggests the stability of the considered structures. When the phonon spectra of unstable structures of $\mathrm{B}_{2} \mathrm{~F}(\mathrm{~d})$ are examined, negative frequencies are the ZA modes, corresponding to the out-ofplane vibrations (see Figures S8 and S9, Supporting Information). Such phonon modes are eliminated for the configuration, which is shown in Figure $2 \mathrm{c}$, by placing $\mathrm{F}$ atoms along a zigzag direction at alternate sites that reconfigures $\mathrm{B}$ atoms along the corrugated direction. We also examine a lower level of coverage, namely, $\mathrm{B}_{4} \mathrm{~F}(\mathrm{~d})$ structure $(\Theta=25 \%)$, but phonon spectrum analysis indicates that this configuration is not stable (see Figure S12, Supporting Information). In a similar manner, BF phases obtained upon structural transition (see above) are not stable configurations (see Figures S10 and S11, Supporting Information).

Following the phonon analyses, we tested the thermodynamic stability of the structures at finite temperatures by using AIMD calculations. We consider a $6 \times 3$ supercell and perform AIMD calculations at increasing temperatures starting from 200 $\mathrm{K}$ and reaching up to $1000 \mathrm{~K}$ in 2.5 ps total simulation time. The snapshots from AIMD simulations are shown in Figure $2 \mathrm{~d}-\mathrm{f}$. As expected, bare borophene preserves its initial structure at high temperatures. Similarly, both $\mathrm{B}_{4} \mathrm{~F}(\mathrm{~s})$ and $\mathrm{B}_{2} \mathrm{~F}(\mathrm{~d})$ remain stable exempting the small fluctuations due to the temperature effect (see Figure 2e,f). Furthermore, neither cluster formations nor local defects in the structures occur, which also indicates the stability of fluorinated derivatives of borophene even at high temperatures. Finally, AIMD results reveal that the BF phase with the honeycomb phase is not stable even at $200 \mathrm{~K}$, although the softening in the phonon dispersion has been reduced (see Figure S10, Supporting Information). These results point out that a stable BF structure similar to borophane could not be obtained as fluorination results in drastic structural alternations leading to phase transitions (see above) which induce instability at ambient conditions. It should be also noted that our results do not fully exclude the possibility of obtaining BF with different phases and/or geometries. Various unstable configurations of halogenated derivatives of monoelement 2D systems are also reported, ${ }^{30,31,51}$ indicating that the stability strongly depends on the geometry, structure, and level of coverage.

Mechanical Properties. After revealing the stable configurations, the mechanical properties of pristine and fluorinated borophene structures $\left[\mathrm{B}_{4} \mathrm{~F}(\mathrm{~s})\right.$ and $\left.\mathrm{B}_{2} \mathrm{~F}(\mathrm{~d})\right]$ are investigated.
First, the elastic tensors are computed and nonzero elastic constants $\left(c_{11}, c_{12}, c_{22}\right.$, and $\left.c_{66}\right)$ are listed in Table 2 . The elastic tensors are then used to calculate the mechanical response of the system, namely, Young's modulus $(Y)$, shear modulus $(G)$, and Poisson's ratio $(\nu)$. Young's moduli of pristine borophene along $\mathbf{a}$ and $\mathbf{b}$ are calculated as $Y_{a}=376.21 \mathrm{~N} / \mathrm{m}$ and $Y_{b}=$ $149.45 \mathrm{~N} / \mathrm{m}$, respectively, and they are in good agreement with previously reported results. ${ }^{9,44,52}$ Interestingly, for both $\mathrm{B}_{4} \mathrm{~F}(\mathrm{~s})$ and $\mathrm{B}_{2} \mathrm{~F}(\mathrm{~d})$, while $Y_{a}$ dramatically decreases, an increase in $Y_{b}$ is noticed. The amount of change is proportional with $\Theta$. When compared to bare borophene, $d_{\mathrm{B}-\mathrm{B}}^{\mathrm{a}}$ increases in $\mathrm{B}_{4} \mathrm{~F}(\mathrm{~s})$ and becomes $1.82 \AA$. This elongation in bond length results in the reduction of $Y_{a}$. In a similar manner, for $\mathrm{B}_{2} \mathrm{~F}(\mathrm{~d}), d_{\mathrm{B}-\mathrm{B}}^{\mathrm{a}}$ is also elongated and becomes 1.75 and $1.84 \AA$ A Nonuniformity in addition to elongation further reduces $Y_{a}$. On the other hand, $d_{\mathrm{B}-\mathrm{B}}^{\mathrm{b}}$ becomes nonuniform upon fluorination and calculated as 1.72 and $2.05 \AA$ for $\mathrm{B}_{4} \mathrm{~F}(\mathrm{~s})$ and $1.68,1.82$, and $2.41 \AA$ for $B_{2} F(d)$. The contraction of $B-B$ bonds corresponding to $B$ atoms without $\mathrm{F}$ induces an increase in $Y_{b}$. It should be noted that anisotropy in mechanical response still remained. When compared with $\mathrm{BH}$, hydrogenation leads to a reduction in $Y$ along both directions unlike fluorination as no contraction in bond lengths is obtained. ${ }^{52} G$ also decreases with increasing $\Theta$ in parallel with hydrogenation; however, the amount of reduction in $\mathrm{B}_{n} \mathrm{~F}$ is less drastic than $\mathrm{BH}$. A summary of the mechanical properties of the currently studied systems and the available data in literature are presented in Table S2 (Supporting Information) for comparison.

Similar to phospherene, ${ }^{53}$ borophene also has a negative Poisson's ratio $(\nu) .{ }^{9,52}$ Consistent with previous theoretical and experimental studies, we obtain negative $\nu_{a}$ and $\nu_{b}$ values. As in the case of $\mathrm{BH}$, interestingly, $\nu$ becomes positive along both directions upon fluorination. While $\nu_{b}$ remains constant at 0.05 , $\nu_{a}$ decreases from 0.07 to 0.03 for $\mathrm{B}_{4} \mathrm{~F}(\mathrm{~s})$ and $\mathrm{B}_{2} \mathrm{~F}(\mathrm{~d})$, respectively. The decrease in $\nu_{a}$ with increasing $\Theta$ can be correlated with structural deformations along the corrugated direction. Last, when compared, the calculated values for $\nu$ are smaller than that reported for borophane.

Electronic Properties. Next, we examine the electronic properties of bare borophene and $\mathrm{B}_{n} \mathrm{~F}$. The resulting electronic band structures are presented in Figure 3. Our results indicate that pristine borophene is metallic along the $\Gamma-X$ and $S-Y$ directions, which are parallel to a. On the other hand, buckling along $\mathbf{b}$ results in energy differences of 4.24 and $9.63 \mathrm{eV}$ along the $Y-\Gamma$ and $X-S$ directions, respectively (Figure 3a). These results are in good agreement with the literature, confirming the strong anisotropy in electronic properties. ${ }^{9,14}$ We find that a low level of $\mathrm{F}$ coverage does not alter the metallic behavior of borophene; however, anisotropy in electronic conduction can be altered. ${ }^{43}$ For $\mathrm{B}_{4} \mathrm{~F}(\mathrm{~s})$, while metallic behavior is retained, energy differences between the band levels in the vicinity of Fermi level significantly decrease compared to its bare counterpart (Figure $3 \mathrm{~b}$ ). This will affect the possible interband transitions and can be interesting for the optical transitions in the mid-/near-infrared and visible spectral range. On the other 

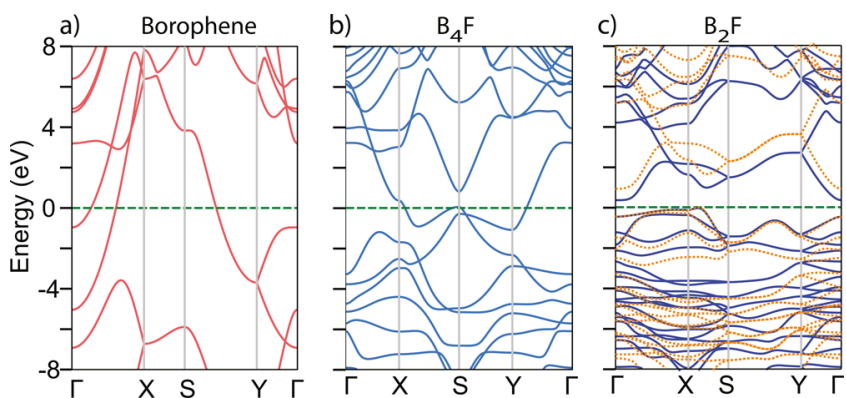

Figure 3. Electronic band structures of (a) bare borophene, (b) $\mathrm{B}_{4} \mathrm{~F}(\mathrm{~s})$, and $(\mathrm{c}) \mathrm{B}_{2} \mathrm{~F}(\mathrm{~d})$. HSE06 results are shown by orange, dotted lines. The Fermi level is set to $0 \mathrm{eV}$ and shown by dashed, green lines.

hand, in $B_{2} F(d)$, the metal to semiconductor transition is obtained with an indirect band gap opening of $0.40 \mathrm{eV}$ between the $X-S$ path and the $\Gamma$-point, as shown in Figure $3 c$. The metal to semiconductor transition can be explained by the saturation of $\mathrm{p}_{z}$ orbitals of $\mathrm{B}$ atoms (occupation of out-of-plane bonding states) upon fluorination. In line with the tendency of borophene to donate electrons, Bader charge analysis confirms the charge transfer from $\mathrm{B}$ to $\mathrm{F}$ atoms upon adsorption. The accepted charges by $\mathrm{F}$ atoms are calculated as $0.81 \mid \mathrm{el}$ and $0.78 \mid \mathrm{el}$ for $\mathrm{B}_{4} \mathrm{~F}$ and $\mathrm{B}_{2} \mathrm{~F}$, respectively. The calculated values are larger than the case of $\mathrm{BH}$ where $0.72 \mathrm{lel}$ is transferred to $\mathrm{H}$ atoms. ${ }^{46}$ The charge distribution analysis also confirms the charge localization on $\mathrm{F}$ atoms and ionic nature of the $\mathrm{B}-\mathrm{F}$ bond. ${ }^{43,54}$ As electronic band gaps are underestimated at the density functional theory-generalized gradient approximation (DFTGGA) level, we repeat the electronic band structure calculations by the HSE06 hybrid functional. While the metallic band structures are not modified, the indirect band gap of $\mathrm{B}_{2} \mathrm{~F}(\mathrm{~d})$ is corrected to $0.89 \mathrm{eV}$. As the electronic structure of $\mathrm{B}_{n} \mathrm{~F}$ strongly depends on the structure, different geometries of coverage and tensile/compressive strain can lead to an indirect to a direct band gap feature. When compared with borophane, $\mathrm{BH}$ is semimetallic with asymmetric Dirac cones. Most of the states around the Fermi level of borophene are removed after hydrogenation, and the remaining states originating from the in-plane $p_{x}$ and $p_{y}$ orbitals lead to band states with linear dispersion. ${ }^{46,48-50}$ However, for $\mathrm{B}_{n} \mathrm{~F}$, the out-of-plane $p_{z}$ orbitals dominate the states around the Fermi level leading to metallic and semiconducting states instead of Dirac cones.

Thermal Properties. In the final section, we present the results on the thermal properties of bare and fluorinated borophene. $2 \mathrm{D}$ materials possess novel thermal properties and have shown a great potential for various applications including thermoelectric energy generation. Thermal rectification, negative thermal resistance, and tunable thermal conductivity are reported for $2 \mathrm{D}$ systems and their heterostructures. ${ }^{55-58}$ For borophene, a very low anisotropic thermal conductivity (14.3 $\mathrm{W} / \mathrm{mK}$ ) which could be useful for thermoelectric and thermal insulation applications is reported. ${ }^{12,13}$ Additionally, remarkable negative thermal expansion coefficients are calculated along both directions. ${ }^{12}$ In this section, the specific heat values at a constant volume $\left(C_{V}\right)$ and entropy $(S)$ are calculated using the expressions $^{59}$ which are summarized in the Supporting Information. For the sake of comparison, the results of graphene are also presented in the Supporting Information, and the obtained quantities are found to be in agreement with the theoretical results and experimental data. ${ }^{60}$
As expected, $C_{V}$ increases with temperature and converges to a constant value of $24 \mathrm{~J} \mathrm{~K}^{-1} \mathrm{~mol}^{-1}$ for both pristine borophene and $\mathrm{B}_{n} \mathrm{~F}$, as shown in Figure 4. This limiting value is higher than

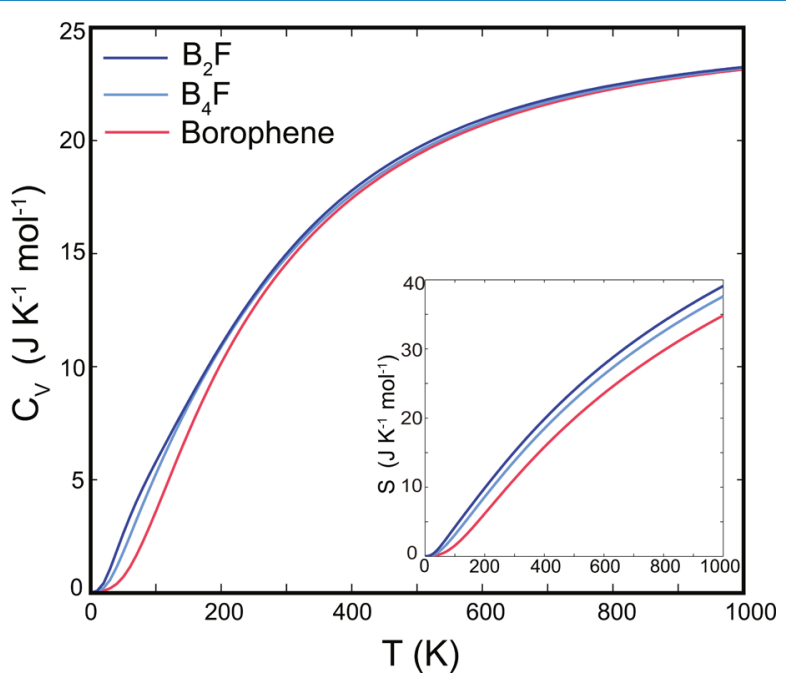

Figure 4. Variation of specific heat at a constant volume $\left(C_{V}\right)$ with temperature for bare borophene, $\mathrm{B}_{4} \mathrm{~F}(\mathrm{~s})$, and $\mathrm{B}_{2} \mathrm{~F}(\mathrm{~d})$. The inset shows the variation of entropy $(S)$.

that obtained for graphene (see Figure S13, Supporting Information). ${ }^{60} C_{V}$ for bare borophene at room temperature is slightly lower than those of $\mathrm{B}_{4} \mathrm{~F}(\mathrm{~s})$ and $\mathrm{B}_{2} \mathrm{~F}(\mathrm{~d})$. Depending on $\Theta, C_{V}$ slightly increases at low temperatures in parallel with the increase in entropy. Higher $C_{V}$ of $\mathrm{B}_{n} \mathrm{~F}$ than bare borophene at low temperatures can be due to the higher phonon density of states that arise by the weak coupling between $\mathrm{F}$ and $\mathrm{B}$ atoms rather than the stiff bonds between $\mathrm{B}$ atoms.

\section{CONCLUSIONS}

In summary, we investigated the possible fluorinated derivatives of borophene and explored their mechanical, electronic, and thermal properties. We showed that $\mathrm{F}$ atoms strongly bind on top of B atoms in the upper layer and adsorption is an exothermic process. Revealing the interaction of a single $\mathrm{F}$ atom with borophene, we studied the possible $\mathrm{B}_{n} \mathrm{~F}(n=1,2$, and 4$)$ structures considering single- and double-sided coverages. The stability analyses performed by a phonon spectrum and hightemperature AIMD calculations indicated that while $\mathrm{B}_{4} \mathrm{~F}$ (25\% coverage $)$ and $\mathrm{B}_{2} \mathrm{~F}(50 \%$ coverage $)$ resulted in stable configurations, $\mathrm{BF}$ ( $100 \%$ coverage) could not be obtained. A full coverage together with applying a tensile strain led to a geometric transition from a triangular to hexagonal or a rectangular borophene structure. However, this BF phases could not remain stable at ambient temperatures. When compared with pristine borophene, the Young's modulus of $\mathrm{B}_{n} \mathrm{~F}$ drastically decreases (up to $70 \%$ ) along the uncorrugated adirection, on the other hand increases (although less significantly) along the corrugated b-direction. In addition, a switch from a negative to positive Poisson's ratio was obtained upon fluorination. We showed that while the metallic character is maintained at low levels of coverage, increasing the $F$ concentration resulted in a metal to semiconductor transition. Finally, the fluorination of borophene increased the specific heat with respect to bare borophene at low temperatures. Our results revealed stable fluorinated derivatives of borophene with versatile properties and enhanced stability, suggesting the $\mathrm{B}_{n} \mathrm{~F}$ 
systems as promising new $2 \mathrm{D}$ materials for various nanoscale applications.

\section{COMPUTATIONAL METHODOLOGY}

The first-principles calculations based on $\mathrm{DFT}^{61,62}$ are performed using the Vienna ab initio simulation package (VASP). ${ }^{63}$ We use projector-augmented wave potentials ${ }^{64}$ with $500 \mathrm{eV}$ kinetic energy cutoff, and the exchange-correlation potential is described by the GGA with the Perdew-BurkeErnzerhof (PBE) functional. ${ }^{65}$ The energy band gap, which is underestimated in this scheme, is corrected by the HeydScuseria-Ernzerhof (HSE) hybrid functional, ${ }^{66,67}$ constructed by mixing $25 \%$ of nonlocal Fock exchange with $75 \%$ of PBE exchange and $100 \%$ of PBE correlation energy. The conjugate gradient method is used to optimize the atomic positions and lattice constants without any constraint. The convergence criteria for total energy and atomic forces between sequential steps have been set to $10^{-5} \mathrm{eV}$ and $0.01 \mathrm{eV} / \AA$, respectively. The Brillouin zone for the primitive unit cell has been sampled by using a $25 \times 19 \times 1 \Gamma$-centered Monkhorst-Pack scheme. ${ }^{68}$ For larger supercells, the $k$-point sampling has been scaled accordingly. A vacuum spacing of $15 \AA$ is placed along the nonperiodic axis to eliminate interactions between periodic images.

To test the thermodynamical stability of the obtained structures, a phonon spectrum is calculated by using the density functional perturbation theory ${ }^{69}$ implemented in VASP. ${ }^{70}$ Furthermore, the stability at high temperatures is tested by using AIMD simulations. All AIMD calculations are performed in $6 \times 3$ supercells, with the temperature starting from $200 \mathrm{~K}$ and then gradually being increased up to $1000 \mathrm{~K}$. The time step used for the integration of equations of motion is set to $1 \mathrm{fs}$, and the total time for the simulation is $2.5 \mathrm{ps}$.

The binding energy $\left(E_{\mathrm{b}}\right)$ and formation energy $\left(E_{\mathrm{f}}\right)$ per $\mathrm{F}$ atom are calculated using the following expressions, $E_{\mathrm{b}}=$ $\left[E_{\mathrm{T}}\right.$ (borophene) $\left.+m_{\mathrm{F}} \cdot E_{\mathrm{T}}(\mathrm{F})-E_{\mathrm{T}}\left(\mathrm{B}_{n} \mathrm{~F}\right)\right] / m_{\mathrm{F}}$ and $E_{\mathrm{f}}=\left[m_{\mathrm{F}_{2}}\right.$. $E_{\mathrm{T}}\left(\mathrm{F}_{2}\right)+E_{\mathrm{T}}$ (borophene) - $\left.E_{\mathrm{T}}\left(\mathrm{B}_{n} \mathrm{~F}\right)\right] / m_{\mathrm{F}}$, where $E_{\mathrm{T}}$ (borophene), $E_{\mathrm{T}}(\mathrm{F}), E_{\mathrm{T}}\left(\mathrm{F}_{2}\right)$, and $E_{\mathrm{T}}\left(\mathrm{B}_{n} \mathrm{~F}\right)$ are the total energies of pristine borophene, single $\mathrm{F}$ atom, $\mathrm{F}_{2}$ molecule, and fluorinated borophene (at different compositions), respectively. $m_{\mathrm{F}}$ and $m_{\mathrm{F}_{2}}$ are the number of $\mathrm{F}$ atoms and $\mathrm{F}_{2}$ molecules, respectively. Accordingly, $E_{\mathrm{b}}>0$ indicates that the adsorption is energetically favorable, and $E_{\mathrm{f}}>0$ implies that fluorination is an exothermic process.

\section{ASSOCIATED CONTENT}

\section{S Supporting Information}

The Supporting Information is available free of charge on the ACS Publications website at DOI: 10.1021/acsomega.7b01730.

Possible adsorption sites and energetics for different levels of $\mathrm{F}$ coverages, phonon dispersions for unstable $\mathrm{B}_{n} \mathrm{~F}$ systems, comparison of mechanical properties with the literature, and equations used to calculate Helmholtz free energy and entropy (PDF)

\section{AUTHOR INFORMATION}

\section{Corresponding Author}

*E-mail: durgun@unam.bilkent.edu.tr (E.D.).

\section{ORCID $\odot$}

Rengin Peköz: 0000-0003-1026-5937

Engin Durgun: 0000-0002-0639-5862

\section{Notes}

The authors declare no competing financial interest.

\section{ACKNOWLEDGMENTS}

The computational resources are provided by TUBITAK ULAKBIM, High Performance and Grid Computing Center (TR-Grid e-Infrastructure) and the National Center for High Performance Computing of Turkey (UHeM) under grant no. 5003622015. This work was supported by the Scientific and Technological Research Council of Turkey (TUBITAK) under project no. 115F088. E.D. acknowledges the financial support from the Turkish Academy of Sciences within Outstanding Young Scientists Award Program (TUBA-GEBIP).

\section{REFERENCES}

(1) Geim, A. K.; Novoselov, K. S. The Rise of Graphene. Nat. Mater. 2007, 6, 183-191.

(2) Bhimanapati, G. R. et al. Recent Advances in Two-dimensional Materials Beyond Graphene. ACS Nano 2015, 9, 11509-11539.

(3) Durgun, E.; Tongay, S.; Ciraci, S. Silicon and III-V Compound Nanotubes: Structural and Electronic Properties. Phys. Rev. B: Condens. Matter Mater. Phys. 2005, 72, 075420.

(4) Vogt, P.; De Padova, P.; Quaresima, C.; Avila, J.; Frantzeskakis, E.; Asensio, M. C.; Resta, A.; Ealet, B.; Le Lay, G. Silicene: Compelling Experimental Evidence for Graphenelike Two-dimensional Silicon. Phys. Rev. Lett. 2012, 108, 155501.

(5) De Padova, P.; Quaresima, C.; Ottaviani, C.; Sheverdyaeva, P. M.; Moras, P.; Carbone, C.; Topwal, D.; Olivieri, B.; Kara, A.; Oughaddou, H.; Aufray, B.; Le Lay, G. Evidence of Graphene-like Electronic Signature in Silicene Nanoribbons. Appl. Phys. Lett. 2010, 96, 261905.

(6) Zhu, F.-f.; Chen, W.-j.; Xu, Y.; Gao, C.-l.; Guan, D.-d.; Liu, C.-h.; Qian, D.; Zhang, S.-C.; Jia, J.-f. Epitaxial Growth of Two-dimensional Stanene. Nat. Mater. 2015, 10, 1020-1025.

(7) Li, L.; Yu, Y.; Ye, G. J.; Ge, Q.; Ou, X.; Wu, H.; Feng, D.; Chen, X. H.; Zhang, Y. Black Phosphorus Field-effect Transistors. Nat. Nanotechnol. 2014, 9, 372-377.

(8) Ji, J.; Song, X.; Liu, J.; Yan, Z.; Huo, C.; Zhang, S.; Su, M.; Liao, L.; Wang, W.; Ni, Z.; Hao, Y.; Zeng, H. Two-dimensional Antimonene Single Crystals Grown by van der Waals Epitaxy. Nat. Commun. 2016, 7, 13352.

(9) Mannix, A. J.; Zhou, X.-F.; Kiraly, B.; Wood, J. D.; Alducin, D.; Myers, B. D.; Liu, X.; Fisher, B. L.; Santiago, U.; Guest, J. R.; Yacaman, M. J.; Ponce, A.; Oganov, A. R.; Hersam, M. C.; Guisinger, N. P. Synthesis of Borophenes: Anisotropic, Two-dimensional Boron Polymorphs. Science 2015, 350, 1513-1516.

(10) Feng, B.; Zhang, J.; Zhong, Q.; Li, W.; Li, S.; Li, H.; Cheng, P.; Meng, S.; Chen, L.; Wu, K. Experimental Realization of Twodimensional Boron Sheets. Nat. Chem. 2016, 8, 563-568.

(11) Lee, C.; Wei, X.; Kysar, J. W.; Hone, J. Measurement of the Elastic Properties and Intrinsic Strength of Monolayer Graphene. Science 2008, 321, 385-388.

(12) Sun, H.; Li, Q.; Wan, X. G. First-principles Study of Thermal Properties of Borophene. Phys. Chem. Chem. Phys. 2016, 18, 1492714932.

(13) Xiao, H.; Cao, W.; Ouyang, T.; Guo, S.; He, C.; Zhong, J. Lattice thermal conductivity of borophene from first principle calculation. Sci. Rep. 2017, 7, 45986.

(14) Peng, B.; Zhang, H.; Shao, H.; Xu, Y.; Zhang, R.; Zhu, H. The Electronic, Optical, and Thermodynamic Properties of Borophene from First-principles Calculations. J. Mater. Chem. C 2016, 4, 35923598.

(15) Penev, E. S.; Kutana, A.; Yakobson, B. I. Can Two-dimensional Boron Superconduct? Nano Lett. 2016, 16, 2522-2526.

(16) Feng, B.; et al. Dirac Fermions in Borophene. Phys. Rev. Lett. 2017, 118, 096401. 
(17) Wu, X.; Dai, J.; Zhao, Y.; Zhuo, Z.; Yang, J.; Zeng, X. C. Twodimensional Boron Monolayer Sheets. ACS Nano 2012, 6, 74437453.

(18) Penev, E. S.; Bhowmick, S.; Sadrzadeh, A.; Yakobson, B. I. Polymorphism of Two-dimensional Boron. Nano Lett. 2012, 12, 2441-2445.

(19) Zhou, X.-F.; Dong, X.; Oganov, A. R.; Zhu, Q.; Tian, Y.; Wang, H.-T. Semimetallic Two-dimensional Boron Allotrope with Massless Dirac Fermions. Phys. Rev. Lett. 2014, 112, 085502.

(20) Gao, M.; Li, Q.-Z.; Yan, X.-W.; Wang, J. Prediction of Phononmediated Superconductivity in Borophene. Phys. Rev. B 2017, 95, 024505.

(21) Xiao, R. C.; Shao, D. F.; Lu, W. J.; Lv, H. Y.; Li, J. Y.; Sun, Y. P. Enhanced Superconductivity by Strain and Carrier-doping in Borophene: A First Principles Prediction. Appl. Phys. Lett. 2016, 109, 122604.

(22) Zhang, Z.; Mannix, A. J.; Hu, Z.; Kiraly, B.; Guisinger, N. P.; Hersam, M. C.; Yakobson, B. I. Substrate-induced Nanoscale Undulations of Borophene on Silver. Nano Lett. 2016, 16, 6622-6627.

(23) Mir, S. H.; Chakraborty, S.; Jha, P. C.; Wärnå, J.; Soni, H.; Jha, P. K.; Ahuja, R. Two-dimensional Boron: Lightest Catalyst for Hydrogen and Oxygen Evolution Reaction. Appl. Phys. Lett. 2016, 109, 053903.

(24) Jiang, H. R.; Lu, Z.; Wu, M. C.; Ciucci, F.; Zhao, T. S. Borophene: A Promising Anode Material Offering High Specific Capacity and High Rate Capability for Lithium-ion Batteries. Nano Energy 2016, 23, 97-104.

(25) Wang, J.; Du, Y.; Sun, L. Ca-decorated Novel Boron Sheet: A Potential Hydrogen Storage Medium. Int. J. Hydrogen Energy 2016, 41, 5276-5283.

(26) Sofo, J. O.; Chaudhari, A. S.; Barber, G. D. Graphane: A Twodimensional Hydrocarbon. Phys. Rev. B: Condens. Matter Mater. Phys. 2007, 75, 153401.

(27) Pujari, B. S.; Gusarov, S.; Brett, M.; Kovalenko, A. Single-sidehydrogenated Graphene: Density Functional Theory Predictions. Phys. Rev. B: Condens. Matter Mater. Phys. 2011, 84, No. 041402(R).

(28) Voon, L. C. L. Y.; Sandberg, E.; Aga, R. S.; Farajian, A. A. Hydrogen Compounds of Group-IV Nanosheets. Appl. Phys. Lett. 2010, 97, 163114.

(29) Houssa, M.; Scalise, E.; Sankaran, K.; Pourtois, G.; Afanas'ev, V. V.; Stesmans, A. Electronic Properties of Hydrogenated Silicene and Germanene. Appl. Phys. Lett. 2011, 98, 223107.

(30) Şahin, H.; Ciraci, S. Chlorine Adsorption on Graphene: Chlorographene. J. Phys. Chem. C 2012, 116, 24075-24083.

(31) Şahin, H.; Topsakal, M.; Ciraci, S. Structures of Fluorinated Graphene and Their Signatures. Phys. Rev. B: Condens. Matter Mater. Phys. 2011, 83, 115432.

(32) Gopalakrishnan, K.; Subrahmanyam, K. S.; Kumar, P.; Govindaraj, A.; Rao, C. N. R. Reversible Chemical Storage of Halogens in Few-layer Graphene. RSC Adv. 2012, 2, 1605-1608.

(33) Karlický, F.; Datta, K. K. R.; Otyepka, M.; Zboril, R. Halogenated Graphenes: Rapidly Growing Family of Graphene Derivatives. ACS Nano 2013, 7, 6434-6464.

(34) Gao, N.; Zheng, W. T.; Jiang, Q. Density Functional Theory Calculations for Two-dimensional Silicene with Halogen Functionalization. Phys. Chem. Chem. Phys. 2012, 14, 257-261.

(35) Ma, Y.; Dai, Y.; Niu, C.; Huang, B. Halogenated Twodimensional Germanium: Candidate Materials for Being of Quantum Spin Hall State. J. Mater. Chem. 2012, 22, 12587-12591.

(36) Si, C.; Liu, J.; Xu, Y.; Wu, J.; Gu, B.-L.; Duan, W. Functionalized Germanene as a Prototype of Large-gap Two-dimensional Topological Insulators. Phys. Rev. B: Condens. Matter Mater. Phys. 2014, 89, 115429.

(37) Tang, W.; Sun, M.; Ren, Q.; Wang, S.; Yu, J. Halogenated Arsenenes as Dirac Materials. Appl. Surf. Sci. 2016, 376, 286-289.

(38) Ma, Y.; Dai, Y.; Guo, M.; Niu, C.; Huang, B. Intriguing Behavior of Halogenated Two-dimensional Tin. J. Phys. Chem. C 2012, 116, 12977-12981.

(39) Nair, R. R.; et al. Fluorographene: A Two-dimensional Counterpart of Teflon. Small 2010, 6, 2877-2884.
(40) Sivek, J.; Leenaerts, O.; Partoens, B.; Peeters, F. M. Firstprinciples Investigation of Bilayer Fluorographene. J. Phys. Chem. C 2012, 116, 19240-19245.

(41) Li, S.-s.; Zhang, C.-w.; Zhang, R.-w.; Li, P.; Li, F.; Yuan, M.; Ren, M.-j.; Ji, W.-x.; Wang, P.-j. First-principles Study of AlN Nanosheets with Chlorination. RSC Adv. 2014, 4, 7500-7505.

(42) Zhou, J.; Wang, Q.; Sun, Q.; Jena, P. Electronic and Magnetic Properties of a BN Sheet Decorated with Hydrogen and Fluorine. Phys. Rev. B: Condens. Matter Mater. Phys. 2010, 81, 085442.

(43) Khanifaev, J.; Peköz, R.; Konuk, M.; Durgun, E. The Interaction of Halogen Atoms and Molecules with Borophene. Phys. Chem. Chem. Phys. 2017, 19, 28963-28969.

(44) Wang, H.; Li, Q.; Gao, Y.; Miao, F.; Zhou, X.-F.; Wan, X. G. Strain Effects on Borophene: Ideal Strength, Negative Possion's Ratio and Phonon Instability. New J. Phys. 2016, 18, 073016.

(45) Osborn, T. H.; Farajian, A. A.; Pupysheva, O. V.; Aga, R. S.; Voon, L. C. L. Y. Ab Initio Simulations of Silicene Hydrogenation. Chem. Phys. Lett. 2011, 511, 101-105.

(46) Xu, L.-C.; Du, A.; Kou, L. Hydrogenated Borophene as a Stable Two-dimensional Dirac Material with an Ultrahigh Fermi Velocity. Phys. Chem. Chem. Phys. 2016, 18, 27284-27289.

(47) Tang, H.; Ismail-Beigi, S. Novel precursors for boron nanotubes: the competition of two-center and three-center bonding in boron sheets. Phys. Rev. Lett. 2007, 99, 115501.

(48) Kou, L.; Ma, Y.; Tang, C.; Sun, Z.; Du, A.; Chen, C. Auxetic and Ferroelastic Borophane: A Novel 2D Material with Negative Possion's Ratio and Switchable Dirac Transport Channels. Nano Lett. 2016, 16, 7910-7914.

(49) Padilha, J. E.; Miwa, R. H.; Fazzio, A. Directional Dependence of the Electronic and Transport Properties of 2D Borophene and Borophane. Phys. Chem. Chem. Phys. 2016, 18, 25491-25496.

(50) Wang, Z.; Lü, T.-Y.; Wang, H.-Q.; Feng, Y. P.; Zheng, J.-C. New crystal structure prediction of fully hydrogenated borophene by first principles calculations. Sci. Rep. 2017, 7, 609.

(51) Boukhvalov, D. W.; Rudenko, A. N.; Prishchenko, D. A.; Mazurenko, V. G.; Katsnelson, M. I. Chemical modifications and stability of phosphorene with impurities: a first principles study. Phys. Chem. Chem. Phys. 2015, 17, 15209-15217.

(52) Wang, Z.; Lü, T.-Y.; Wang, H.-Q.; Feng, Y. P.; Zheng, J.-C. High Anisotropy of Fully Hydrogenated Borophene. Phys. Chem. Chem. Phys. 2016, 18, 31424-31430.

(53) Jiang, J.-W.; Park, H. S. Negative Poisson's Ratio in Single-layer Black Phosphorus. Nat. Commun. 2014, 5, 4727.

(54) Ning, F.; Wang, D.; Feng, Y.-X.; Tang, L.-M.; Zhang, Y.; Chen, K.-Q. Strong interfacial interaction and enhanced optical absorption in graphene/InAs and MoS2/InAs heterostructures. J. Mater. Chem. C 2017, 5, 9429-9438.

(55) Chen, X.-K.; Xie, Z.-X.; Zhou, W.-X.; Tang, L.-M.; Chen, K.-Q. Thermal rectification and negative differential thermal resistance behaviors in graphene/hexagonal boron nitride heterojunction. Carbon 2016, 100, 492-500.

(56) Guo, Z.; Zhang, D.; Gong, X.-G. Thermal conductivity of graphene nanoribbons. Appl. Phys. Lett. 2009, 95, 163103.

(57) Chen, X.-K.; Xie, Z.-X.; Zhou, W.-X.; Tang, L.-M.; Chen, K.-Q. Phonon wave interference in graphene and boron nitride superlattice. Appl. Phys. Lett. 2016, 109, 023101.

(58) Chen, X.-K.; Liu, J.; Peng, Z.-H.; Du, D.; Chen, K.-Q. A wavedominated heat transport mechanism for negative differential thermal resistance in graphene/hexagonal boron nitride heterostructures. Appl. Phys. Lett. 2017, 110, 091907.

(59) Togo, A.; Chaput, L.; Tanaka, I.; Hug, G. First-principles Phonon Calculations of Thermal Expansion in Ti3SiC2, Ti3AlC2, and Ti3GeC. Phys. Rev. B: Condens. Matter Mater. Phys. 2013, 87, 174301.

(60) Pop, E.; Varshney, V.; Roy, A. K. Thermal Properties of Graphene: Fundamentals and Applications. MRS Bull. 2012, 37, 1273.

(61) Hohenberg, P.; Kohn, W. Inhomogeneous Electron Gas. Phys. Rev. 1964, 136, B864.

(62) Kohn, W.; Sham, L. J. Self-consistent Equations Including Exchange and Correlation Effects. Phys. Rev. 1965, 140, A1133. 
(63) Kresse, G.; Furthmüller, J. Efficient Iterative Schemes for Ab Initio Total-energy Calculations Using a Plane-wave Basis Set. Phys. Rev. B: Condens. Matter Mater. Phys. 1996, 54, 11169.

(64) Blöchl, P. E. Projector Augmented-wave Method. Phys. Rev. B: Condens. Matter Mater. Phys. 1994, 50, 17953.

(65) Perdew, J. P.; Burke, K.; Ernzerhof, M. Generalized Gradient Approximation Made Simple. Phys. Rev. Lett. 1996, 77, 3865.

(66) Heyd, J.; Scuseria, G. E.; Ernzerhof, M. Hybrid Functionals Based on a Screened Coulomb Potential. J. Chem. Phys. 2003, 118, 8207.

(67) Paier, J.; Marsman, M.; Hummer, K.; Kresse, G.; Gerber, I. C.; Ángyán, J. G. Screened Hybrid Density Functionals Applied to Solids. J. Chem. Phys. 2006, 124, 154709.

(68) Monkhorst, H. J.; Pack, J. D. Special Points for Brillouin-Zone Integrations. Phys. Rev. B: Solid State 1976, 13, 5188.

(69) Baroni, S.; de Gironcoli, S.; Corso, A. D.; Giannozzi, P. Phonons and Related Crystal Properties from Density-functional Perturbation Theory. Rev. Mod. Phys. 2001, 73, 515.

(70) Togo, A.; Tanaka, I. First Principles Phonon Calculations in Materials Science. Scr. Mater. 2015, 108, 1-5. 